\title{
A Critical Evaluation of Direct Electrical Protein Detection Methods*
}

\author{
P. Bergveld \\ University of Twente, Box 217, 7500 AE Enschede, The Netherlands
}

(Received 10 April 1990; revised version received 11 June 1990; accepted 12 June 1990)

\begin{abstract}
During the last decennia many protein-related electrical phenomena have been studied and applied in a variety of measuring systems, from simple metal electrodes with adsorbed proteins to sophisticated systems with lipid bilayers. Many of the investigations concern the monitoring of immuno reactions. The basic underlying electrical effects of the observed phenomena are the protein modulated dielectric constant, conductivity, electrical potential, ion permeability and ion mobility. In this paper special attention is paid to the capacitive measurements with EIS systems as well as impedance and potential measurements with FET devices. The Donnan theory is treated and applied to the static ImmunoFET operation, explaining the relatively small effects which have been reported. Finally, an alternative approach is described in which the ImmunoFET is applied in a dynamic way, to circumvent the drawbacks of the static measurements.
\end{abstract}

Keywords: IMFET, ISFET, biosensor, protein measurement, immunosensor.

\section{INTRODUCTION}

The progressive development of miniature solidstate sensors during the last decades, made possible by the application of modern technologies such as silicon technology, thin and thick film technologies and membrane technologies, has also inspired the development of immuno sensors. Many papers already describe specific combinations of a miniature chemically sensitive electrode, or - even more simply - an electrically sensitive electrode with an immunologically active surface layer or membrane. Because there is no generic way of operating the different types of devices, the various approaches are difficult to review in a constructive sequence.

* Part of this paper was presented at Biosensors 90 , Singapore, 2-4 May 1990.
In order to do so, some of the measurement methods have to be excluded, such as all indirect measurements which make use of radioactive labels, enzymes, etc. for transforming an immunological signal into a chemical one, which is then converted into an electrical signal by means of an appropriate ion or gas sensor. Only those sensing methods will be discussed which explicitly make use of some intrinsic properties of a protein in general, resulting in a directly measurable electrical effect, which can be modulated by the immunochemical reaction of an antigen with its corresponding antibody. In this case the sensor selectivity relies on the selective binding of these species.

The reported electrical effects of the adsorption of a layer of proteins to an electrode surface or of an immuno reaction at a protein precoated electrode surface, sometimes immobilized in a 
membrane, can roughly be divided into two classes: (1) electrode impedance measurements; and (2) electrode potential measurements.

The measuring results can be related in one way or another to the intrinsic protein properties: charge distribution, dimension and shape. These properties may influence the dielectric constant and conductivity of a protein layer and thus the impedance as well as the ion permeability and ionic mobility, and the surface potential.

This paper investigates how different results of measurements reported throughout the years by various research groups are interrelated, and whether they can possibly be explained by a general theory, concentrated on the applied measuring technique as such, irrespective of limitations resulting from equilibrium and kinetic characteristics of binding reactions and from mass transport constraints (Eddowes, 1987).

\section{ELECTRODE IMPEDANCE MEASUREMENTS}

In the literature concerning immuno electrodes, impedance measurements are often reported as capacitive measurements, most probably because it was expected that the adsorption of a layer of proteins would 'insulate' an electrode from the surrounding liquid. Furthermore it was thought that if these proteins were antibodies it should be possible to modulate the related capacitive effect by an immuno reaction. Therefore many electrode capacitances have been measured as a function of protein adsorption, in which case the electrode was either a metal, or insulated metal, or an insulated semiconductor. The most obvious examples of these type of measurements will be described and analysed in the next sections.

\section{Capacitive measurements with metal electrodes}

The application of a simple metal wire, onto which proteins adsorb, should be the most direct way of protein sensing, comparable with many electrochemical sensors such as coated wires, metal oxide/metal electrodes, etc. This is only possible if the frequency response of the electrode can directly be related to the concentration or activity of the adsorbed species. The specific affinity of a precoated layer of proteins should favor the desired selectivity.

Many protein adsorption experiments have been carried out throughout the years with a large variety of substrates. Most noteworthy is the work of the research group of Lundstrom at the University of Linköping, Sweden which focussed, in the 1980s especially, on the sensing properties of adsorbed proteins on metal electrodes. Developing a sensor for the measurement of enzymatic activity, Arwin made use of the fact that certain peptides, which can be hydrolyzed by enzymes, adsorb on a metal electrode, causing a concentration-dependent double-layer capacitance of the electrode (US Patent, 1978; Arwin et al., 1982). The two parts of the peptide which originate from the enzymatic cleavage do not have this property. More specifically, the adsorption of the tripeptide Bz-Phe-Val-ArgpNA (S2160) onto a platinum electrode could be monitored as a decrease of the electrode capacitance, while the subsequent cleavage of S2160, due to added thrombin, resulted in an increase of the electrode capacitance. Figure 1 shows a typical curve of the electrode capacitance as a function of the added substrate and enzyme, redrawn from the corresponding patent description.

For reproducibility of the measurements a carefully performed electrochemical cleaning procedure of the electrodes appeared to be necessary.

As a conclusion it can be said that under specific conditions it seems possible that a metal surface can be insulated electrically by the adsorption of a layer of small organic molecules, while this effect is reversed by desorption. The effect can be measured as a change in the metal/ electrolyte capacitance. Not only adsorption as such, but also a certain molecular orientation, is

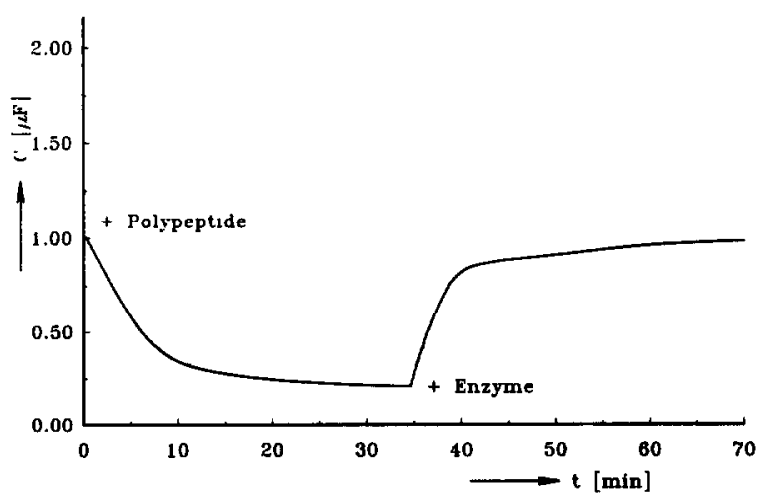

Fig. 1. Measured capacitance as function of time when substrate is added as well as enzyme. (Redrawn from US Patent, 1978.) 
most probably necessary to obtain this effect. Depending on the concentration of the peptide and the measurement frequency, the capacitance is in the order of $10 \mu \mathrm{F} / \mathrm{cm}^{2}$, as compared with $25 \mu \mathrm{F} / \mathrm{cm}^{2}$ for a bare electrode.

At the same Institute (Ivarsson et al., 1981) the titanium/electrolyte interface has been studied upon the adsorption of plasma proteins. Adsorption of human serum fibrinogen (HFIB) and human serum albumin (HSA) onto a titanium electrode resulted in capacitance changes in the order of $1-2 \mu \mathrm{F} / \mathrm{cm}^{2}$, hardly dependent on the type and concentration of the proteins. The effect is much smaller than in the case of peptide adsorption, mentioned in the previous example. Figure 2 shows a typical registration of $\delta \mathrm{C}$ upon the addition of varying concentrations of HFIB and HSA. After a large initial increase upon the first addition of HFIB, $\delta$ C increased only slightly upon further addition of HFBI and upon further additions of HSA (Fig. 2). Similar effects were observed if first HSA and then HFBI were added.

The mechanism of adsorption in relation to the sequence of added proteins is unexplained.

Ellipsometric control shows layer thicknesses in the order of $2 \mathrm{~nm}$ in the case of peptides, as mentioned in the previous example, and of $12 \mathrm{~nm}$ in the case of the plasma proteins. In general, a thicker layer should give a larger decrease in capacitance than a thinner layer, provided that the density and the dielectric constant of the layers are the same. Because this is not observed, but even the opposite effect, the authors conclude that the adsorbed layers of HFIB and HSA are

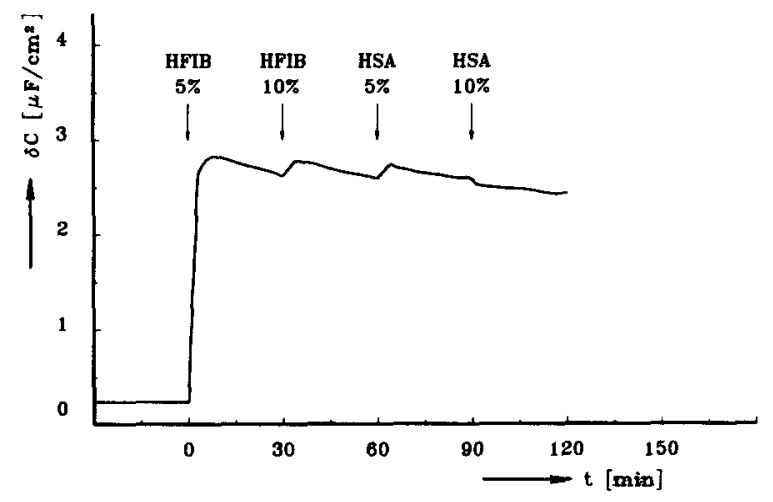

Fig. 2. Measured capacitance change during adsorption of HFIB and HSA on a titanium electrode. The first protein is injected in the sample solution at the starting point and after $30 \mathrm{~min}$. The second protein is added after 60 and $90 \mathrm{~min}$. respectively. (Redrawn from Ivarsson et al., 1981.) much less dense, and thus more porous, than the peptide layers.

In a much more recent study Champagne et al. (1988) reported on preliminary results of capacitance measurements with gold electrodes upon the adsorption of biotin and subsequent surface perturbation, induced by adding avidin to the solution. The adsorption process of biotin appears to be a function of the electrode potential and can thus be controlled by choosing an appropriate potential in relation to the biotin concentration. Applying a constant dc potential to the electrode, its capacitance decreases from 60 to $15 \mu \mathrm{F} / \mathrm{cm}^{2}$ within $3 \mathrm{~min}$ after the addition of biotin. The final steady-state capacitance increases again from 15 to $20 \mu \mathrm{F} / \mathrm{cm}^{2}$ after addition of avidin, within approximately the same time. The latter capacitance change is thought to be caused by the formation of biotin/avidin complexes which desorb from the surface, but which probably do not completely remove the original layer of biotin. A typical registration is shown in Fig. 3.

In a further experiment the biotin was modified by addition of a lauryl chain to its carboxyl group in order to increase its hydrophobic character. This modified biotin appeared to adsorb to the gold electrode in the same way as the unmodified biotin as observed from the change in electrode capacitance. Addition of avidin, however, does not change the capacitance of this modified biotin/gold electrode. Champagne et al. (1988) suggested that the modified biotin/avidin complex does not desorb from the electrode.

The experiments described till now show not only that biological molecules may adsorb to

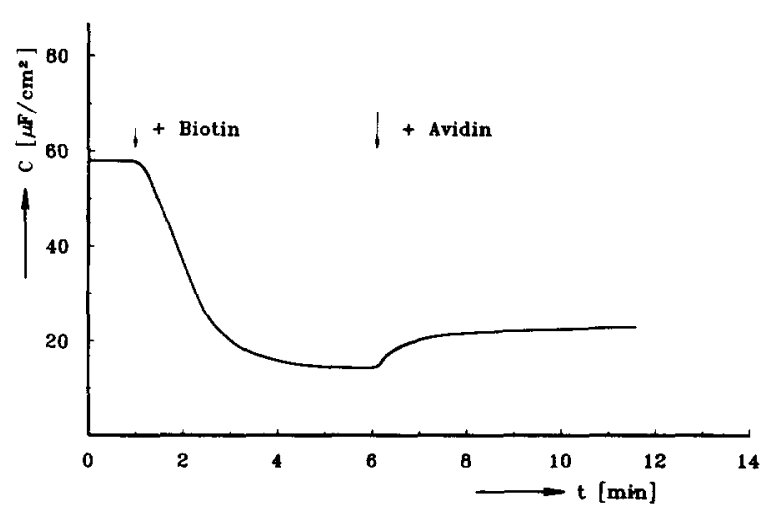

Fig. 3. Variation of the capacitance with time, recorded at constant applied potential a gold electrode $(-0.035 \mathrm{~V} /$ $S C E)$ upon the addition of biotin, and subsequently avidin, to the $01 \mathrm{M} \mathrm{KCl} \mathrm{pH} 4$ solution. (Redrawn from Champagne et al, 1988.) 
metal substrates (which is of course not surprising), but in addition that the adsorbed layer may manifest itself as a dielectric layer between the metal surface and the electrolyte, which can be measured simply as a change in the electrode capacitance.

The question arises, however, whether the measurement of electrode capacitances can distinguish between biological effects such as competition in affinity (avidin/biotin versus biotin/gold or modified biotin/gold) and more physical effects such as the degree of coverage in relation to molecule conformation. A thin dense layer may result in the same change in capacitance as a thicker less dense layer. One should therefore be careful to interpret capacitance measurements in terms of the existence of homogeneous layers which are formed upon adsorption and released upon desorption.

\section{Capacitive measurements with insulated metal electrodes}

A quite separate class of experiments is formed by the capacitive measurements with electrolyte/ insulator/conductor systems, influenced by the adsorption of biological macromolecules. A rather isolated experiment is reported by Newman et al. (1986). A planar capacitive device was constructed, consisting of two interdigitated copper electrodes at a glass substrate, covered with a layer of parylene and silicon monoxide. An antibody-conserving molecular sieve completed the structure. The dielectric constant between the electrodes was determined by means of a capacitance measurement with respect to a reference structure. The measuring device was covered with immobilized Tricothecene toxin $\mathrm{T} 2$. T2-specific antibodies from the analyte may bind to this immobilized hapten, in competition with binding to free hapten. Upon addition of T2-specific antibodies, the capacitance dropped from $2180 \mathrm{pF}$ to $2120 \mathrm{pF}$, while subsequent addition of $\mathrm{T} 2$ resulted in an increase of the capacitance to the original value. A preliminary result is shown in Fig. 4.

Newman et al. (1986) conclude that antibodyantigen reactions, which occur at the device surface, produce significant and reversible capacitance changes on a time scale of several minutes. Unfortunately no further information is given, so that the results cannot be interpreted in relation to concentrations of the background

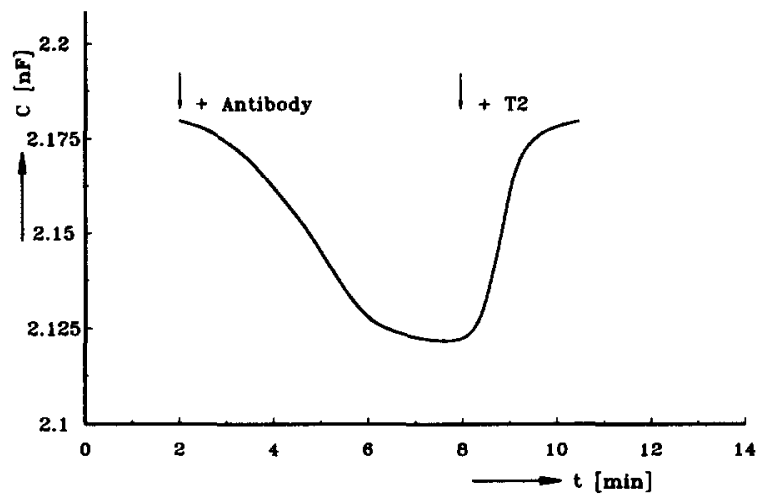

Fig. 4. Variation of the device capacitance upon the addition of $T 2$ specific antibodies and subsequently $T 2$. (Redrawn from Newman et al, 1986.)

electrolyte and the analyte, nor other physical and chemical environmental conditions.

In general it can be stated that, for all capacitive measurements described up to now, the information obtained is limited if no additional data are available, such as layer thicknesses and other physical and chemical parameters. With respect to this limitation the capacitive measurements at electrolyte/insulator/ semiconductor systems (EIS) are much more useful, especially regarding the so-called capacitance-voltage (C-V) measurements. This class of measurements will therefore be treated in more detail.

\section{Capacitive measurements with electrolyte/ insulator/semiconductor systems}

Capacitance-voltage measurements with biologically modified EIS systems originate from the ambition to construct an ImmunoFET for the direct measurement of an immuno reaction (Bergveld \& Sibbald, 1988). From the C-V measurements, however, more information can be withdrawn than from the static FET measurements which will be treated in a later section. Therefore first of all the significance of $\mathrm{C}-\mathrm{V}$ measurements will be explained in detail, while afterwards the various experimental results as reported in the literature will be reviewed and discussed.

By applying an ac voltage to the EIS system as schematically given in Fig. 5, the capacitance of the structure can be measured, similar to the wellknown measurements with MOS capacitances (Bergveld \& Sibbald, 1988). 


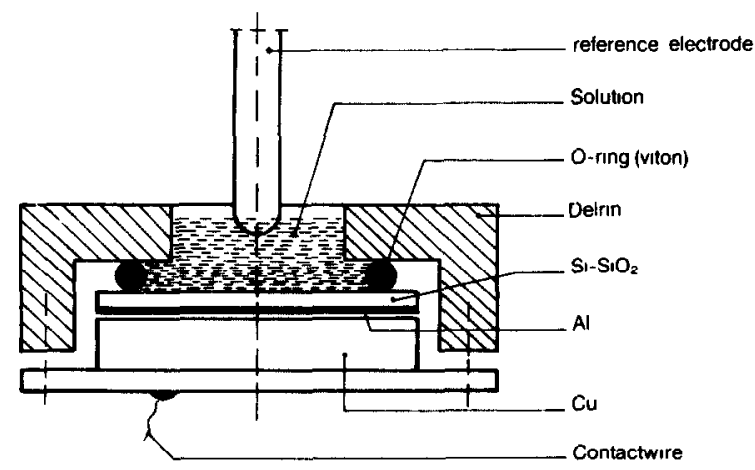

Fig. 5. Schematic representation of the sample mounting of an electrolyte-SiO${ }_{2}-\mathrm{Si}$ structure for $\mathrm{C}-\mathrm{V}$ measurements.

The capacitance $C$, measured at a certain frequency, can be considered as resulting from a series connection of the capacitance $C_{\mathrm{ox}}$ of the insulator, usually silicon dioxide, and the capacitance $C_{\mathrm{Si}}$, the space-charge capacitance of the silicon.

$$
C=\frac{C_{\mathrm{ox}} C_{\mathrm{Si}}}{C_{\mathrm{ox}}+C_{\mathrm{Si}}}
$$

If $C_{\mathrm{ox}} \gg C_{\mathrm{Si}}$, then $C=C_{\mathrm{Si}}$, while if $C_{\mathrm{Si}} \gg C_{\mathrm{ox}}$, then $C=C_{\mathrm{ox}}$. In eqn (1) $C_{\mathrm{ox}}$ has a constant value, determined by the dielectric constant and the thickness of the insulating layer, while $C_{\mathrm{Si}}$ depends on the thickness of the depletion layer in the silicon, which is among others a function of the applied dc voltage. The relation between the capacitance $C$, measured at a relatively low frequency $(100 \mathrm{~Hz})$, and the applied dc voltage, usually provided by a ramp generator, is given in Fig. 6, curve (a).

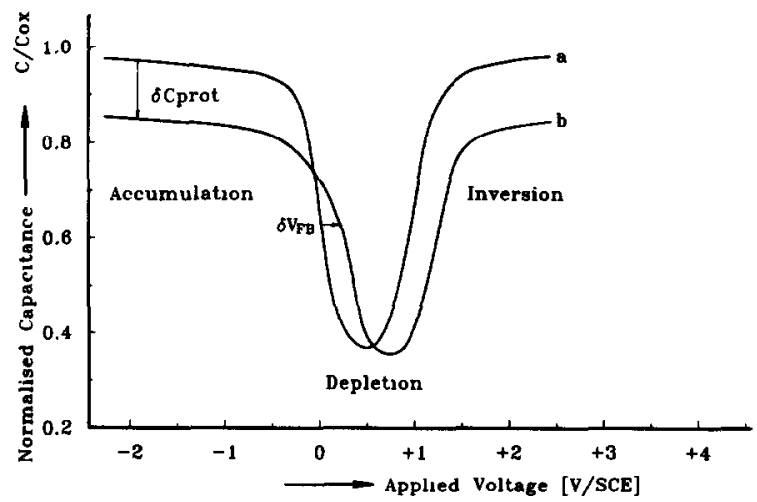

Fig. 6. Typical C-V curve. Curve (a), bare EIS sample; curve (b), EIS sample with adsorbed proteins, assuming $\left(\mathrm{Q}_{o x}+\mathrm{Q}_{\mathrm{s} 0}\right)=0$.
Three regions in this so-called $\mathrm{C}-\mathrm{V}$ curve can be distinguished: the accumulation, the depletion and the inversion regions. This can be explained as follows. With a p-type silicon substrate (as an example), a large negative potential applied to the electrolyte will cause accumulation of holes at the silicon surface. The measured capacitance now approaches $C_{\text {ox }}$. When the negative bias is low, the space charge variations due to the measuring signal (a low amplitude ac signal superimposed upon the dc bias) no longer occur close to the interface. Consequently, $C_{\mathrm{Si}}$ may become comparable to $C_{\mathrm{ox}}$, and the measured capacitance $C$ will be smaller than $C_{\text {ox }}$. When the de voltage is further increased, a complete depletion layer is formed at the silicon surface, and the measured capacitance will approach the value of $C_{\mathrm{Si}}$. At a sufficiently high, positive voltage, an inversion layer of free electrons is formed at the surface and the measured capacitance again becomes equal to the insulator capacitance $C_{\text {ox }}$.

An $n$-type silicon substrate shows identical $\mathrm{C}-\mathrm{V}$ curves, but the polarity of the voltages is reversed: a positive voltage causes accumulation and a negative voltage inversion.

The position of the $\mathrm{C}-\mathrm{V}$ curve along the voltage axis is influenced by several solid-state parameters such as the silicon electron work function: $\boldsymbol{\Phi}_{\mathrm{Si}}$, the charges located in the oxide: $Q_{o x}$ and in surface and interface states: $Q_{\text {ss }}$ and by the electrochemical parameters $E_{\text {ref, }}$, the reference electrode potential and $\left(\psi_{0}-\chi^{\mathrm{sol}}\right)$, the electrolyteoxide interfacial potential. These influences are gathered in the so-called flat band voltage of the silicon

$$
V_{\mathrm{FB}}=E_{\mathrm{ref}}-\psi_{0}+\chi^{\mathrm{sol}}-\frac{\Phi_{\mathrm{Si}}}{q}-\frac{Q_{\mathrm{ox}}+Q_{\mathrm{ss}}}{C_{\mathrm{ox}}}
$$

The parameter $\psi_{0}$ is a function of the $\mathrm{pH}$ of the electrolyte and is therefore the chemical input variable on which the ISFET operation is based (Bergveld \& Sibbald, 1988).

For the present the charges $Q_{\mathrm{ox}}+Q_{\mathrm{ss}}$ will be assumed to be zero in order to exclude the influence of $C_{\mathrm{ox}}$ on the value of $V_{\mathrm{FB}}$. This value can then be calculated and appears to be for a p-type MOS capacitance $-0.6 \mathrm{~V}$ and for an analogous EIS capacitance at $\mathrm{pH}=70 \mathrm{~V}$. A shift of the $\mathrm{C}-\mathrm{V}$ curve along the $\mathrm{V}$-axis is characterized by a corresponding $\delta V_{\mathrm{FB}}$, as illustrated in Fig. 6 .

If at the oxide/electrolyte interface a dense layer of proteins adsorbs, an additional capacitance may manifest itself in series with the device 
capacitance, similar to the silicon depletion capacitance $C_{\mathrm{Si}}$, which appears in series with $C_{\mathrm{ox}}$ at a certain bias voltage. Assuming that an eventual series capacitance $C_{\text {prot }}$ is independent of the bias voltage, it will shift the original $\mathrm{C}-\mathrm{V}$ curve along the $C$-axis over the full voltage range, but relatively more in the accumulation and inversion regions than in the depletion region. The resulting $\mathrm{C}-\mathrm{V}$ curve is schematically drawn in Fig. 6, curve (b). As can be seen in Fig. 6, curve (b) is also shifted along the voltage axis with respect to curve (a), indicating that the adsorbed layer of proteins may also induce an interfacial potential at the electrolyte side of the layer, thus in series with the applied voltage. The simultaneously occurring shift of the $\mathrm{C}-\mathrm{V}$ curve along the capacitance as well as along the voltage axis, $\delta C_{\text {prot }}$ and $\delta V_{\mathrm{FB}}$ respectively, makes the $\mathrm{C}-\mathrm{V}$ measurement more interesting than the capacitance measurements on metal surfaces as dealt with in the previous section. The information that can be withdrawn from one and the same measurement is twice as much if the two effects are independent, which is the case if the condition is satisfied that $\left(Q_{o x}+Q_{s s}\right)=0$. Both effects should therefore be considered and discussed when reviewing the literature concerning measurements of $\mathrm{C}-\mathrm{V}$ curves as a function of protein adsorption or immuno reactions.

The Swedish research group, already mentioned in the previous section, was one of the first to investigate the influence of protein adsorption on silicon dioxide by means of $\mathrm{C}-\mathrm{V}$ measurements in comparison with ellipsometric determination of layer thicknesses (Stenberg et al., 1979). By using samples with different thicknesses of the oxide layer the degree of coverage could be evaluated. The capacitance of the system was measured in the accumulation region and studied as function of frequency. Upon the addition of $0.5 \mathrm{mg} / \mathrm{ml}$ bovine serum albumin (BSA), the ellipsometric measurement showed that a $1.6 \mathrm{~nm}$ thick layer had been adsorbed after approximately $100 \mathrm{~min}$. However, only a small shift in the capacitance $(1.5 \%)$ was observed. The authors conclude that the protein molecules probably only have a small contact area with the substrate and hence are not able to insulate the surface completely.

A different behaviour was found for samples where a tripeptide (see previous section) was adsorbed. The layer thickness was $29 \mathrm{~nm}$, as determined ellipsometrically, and the capacitance changed from 0.6 to $0.8 \mu \mathrm{F} / \mathrm{cm}^{2}$, slightly influenced by the frequency of measurement. When the peptide layer was enzymatically destroyed by thrombin, the capacitance shifted back to $0.75 \mu \mathrm{F} / \mathrm{cm}^{2}$. The conclusion was drawn that the peptide gives a more dense film than BSA, but has fewer insulating properties after the enzymatic cleavage.

In the experiments described above, no information is given concerning a possible shift of the $\mathrm{C}-\mathrm{V}$ curve along the voltage axis. Only upon the adsorption of a monolayer of a lipid (behenic acid) was a decrease of the capacitance observed as well as a shift of $200-400 \mathrm{mV}$ along the voltage axis. The authors concluded that it is most probably only in this case that the film has an impedance high enough to measure a potential. In other films the introduction of channelforming agents strongly affected the measurements. This was explained by the appearance of small, conducting channels or pores, which drastically influence the measurements. These observations and considerations show that the interpretation of $\mathrm{C}-\mathrm{V}$ curves upon adsorption of organic molecules is more difficult than originally thought.

A more recent and most interesting study concerning capacitive measurements with an EIS system has been published (Bataillard et al., 1988; Gardies et al., 1989) as a basic feasibility study for the direct detection of immunospecies. The silicon/silicon dioxide samples were carefully pretreated (hydration of the silica layer). In order to obtain a well-oriented layer of amino groups for the covalent coupling of proteins, a monofunctional silane (4-amino-butyl dimethylmethoxisilane) was used, resulting in a very high degree of coverage. Measurements were carried out at $10 \mathrm{kHz}$, resulting in so-called highfrequency $\mathrm{C}-\mathrm{V}$ curves, as shown in Fig. 7. Because the potentials are defined with opposite polarity, the curves are reversed along the voltage axis with respect to the curves given in Fig. 6, while in the inversion region the capacitance remains low because at high frequencies the free electrons cannot follow the applied ac signal. The curve marked 'oxide' is of a bare sample. After silanizing the oxide, the curve marked 't silane' was obtained, which shows a decrease in capacitance in the accumulation region, indicating the existence of an additional dielectric layer on top of the oxide.

As a next step antibodies were immobilized to 


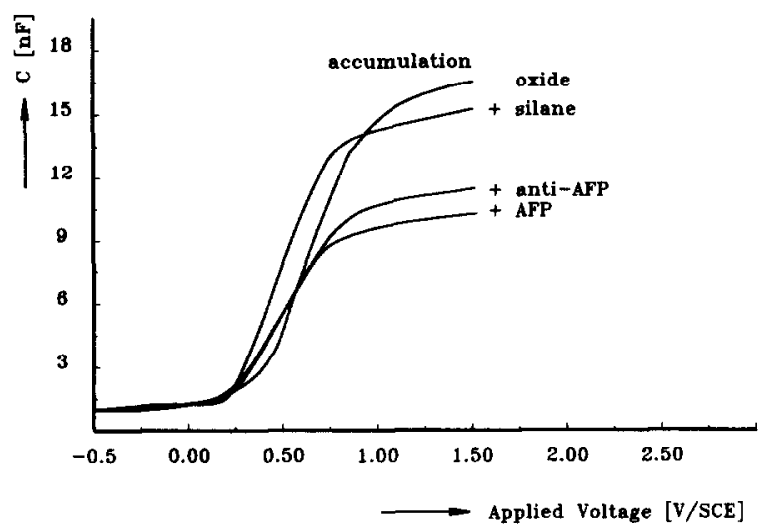

Fig. 7. $\mathrm{C}-\mathrm{V}$ Curves of $\mathrm{Si} / \mathrm{SiO} \mathrm{O}_{2}$ samples measured by Bataillard et al (1988): blank, after silanization and after adsorption of anti-a-fetoprotein and subsequent reaction with a-fetoprotein. (Redrawn from Bataillard et al. (1988).)

the silanized surface by a glutaraldehyde procedure, specifically monoclonal anti-afetoprotein. As can be seen from curve '+ antiAFP' in Fig. 7, the capacitance decreased again. After $42 \mathrm{~min}$ of incubation of serum, containing $400 \mathrm{ng} / \mathrm{ml}$ of $\alpha$-fetoprotein, the final $\mathrm{C}-\mathrm{V}$ curve was recorded, showing a further decrease of the capacitance (curve '+ AFP').

At first sight neither the initial protein adsorption nor the immuno reaction result in a voltage shift of the curve but only in a change in capacitance.

Considering these results, the authors concluded that the capacitance measurement is suitable for the direct detection of an antibodyantigen interaction, but, because no change in $V_{\mathrm{FB}}$ is observed, they also concluded that a related measurement with a field effect transistor - in which case a voltage shift is explicitly measured - will not result in any signal.

However, it must be stated here that this conclusion is not correct. In the previous theoretical considerations it was assumed that no solid-state charges were present in the system $\left(Q_{\mathrm{ox}}+Q_{\mathrm{ss}}=0\right)$. However, if this is not the case, eqn (2) predicts that a decrease of the capacitance will always coincide with a simultaneous increase of the flat band voltage $V_{\mathrm{FB}}$, independent of any other additional interfacial potential. This effect is consistent with that observed after silanization. The curve denoted as ' + silane' shows a decrease of the capacitance accompanied by a shift of the curve to the left. It may thus be concluded that the samples are not free of oxide and/or interface charges, which conclusion can also be drawn from the observed positive value of $V_{\mathrm{FB}}$. This means that a further decrease of the capacitance (curves + anti-AFP and subsequently + AFP) should coincide with a further shift of the $C-V$ curve to the left, but because this is not observed the conclusion should be that a compensating interfacial potential must be present, shifting the $\mathrm{C}-\mathrm{V}$ curve back to the right. This conclusion means that the experiments of Bataillard et al. show that the adsorption of a protein layer at the surface of an oxide manifests itself as a change in capacitance as well as in interfacial potential. The value of this potential can, however, not be derived from the $\mathrm{C}-\mathrm{V}$ measurements, because the vertical and the lateral shift of the $C-V$ curves are not independent due to the presence of oxide and/or interface charges. Furthermore the origin of a compensation effect is unknown, although it may be feasible that the appearance of an interfacial potential will coincide with an adaptation of the $\mathrm{pH}$ inside the protein layer, which will modulate the value of $\psi_{0}$ (eqn (2)). This effect may be based on the Donnan phenomenon, as will be explained in detail in a later section.

The modulation of $\psi_{0}$ by adsorbed biological molecules may also be present in the $\mathrm{C}-\mathrm{V}$ measurements of Grattarola et al. (1989). In this case C-V curves of $\mathrm{Si}_{-} \mathrm{Si}_{3} \mathrm{~N}_{4}$ samples, similar to the ones described above, have been measured but now with a coating of laminin, a compound that is often utilized in biological research for improving cell adhesion to substrates. In contrast to the C-V curves measured by Bataillard et al., no change in the capacitance was observed after the addition of laminin, but the $\mathrm{pH}$ sensitivity of the samples decreased and, although not very reproducible, a very small shift of the flat-band voltage towards negative values was reported. Because no capacitive effect was observed, it may be concluded that most probably the density of the laminim coating is much less than that of the anti-AFP/AFP layers, but that the interfacial potential $\psi_{0}$ is influenced in one way or a nother.

That a protein coating may create microchemical surroundings with a composition which deviates from the bulk electrolyte composition is proven by Schasfoort et al. (1989a), however, not by means of a $\mathrm{C}-\mathrm{V}$ measurement, but with an ISFET measurement, as will be described in the next section. 


\section{IMPEDANCE MEASUREMENTS WITH AN ISFET, MODIFIED BY BIOLOGICAL MATERIALS}

Schasfoort et al. (1989a) investigated the influence of an HSA/anti-HSA precipitate, deposited on the surface of an ISFET, on the ISFET behaviour. With respect to the static behaviour, it was found that no change in $\psi_{0}$ occurred when compared with a bare ISFET, but that only the response time, upon a step in $\mathrm{pH}$, increased with increasing thickness of the precipitate layer, being also larger for lower buffer capacities of the background electrolyte. The conclusion was that the protein layer limited the diffusion time of ions, resulting in the observed larger response time, but that the $\mathrm{pH}$ sensitivity was not influenced.

In contrast to what has been found with respect to the dc behaviour, the ac behaviour of an ISFET is very much influenced by the presence of a protein layer. The measurement of the frequency response of an ISFET precoated with a protein precipitate initially showed a higher characteristic $-3 \mathrm{~dB}$ point than that of the bare ISFET. However, this point decreased with time towards a final equilibrium point, which nevertheless appeared to be still higher than that of the bare ISFET. This effect is shown in Fig. 8, curves (1) to (11), where curve (0) is of the bare ISFET.

The final $-3 \mathrm{~dB}$ point appeared to be a function of $\mathrm{pH}$. The conclusion was drawn that the ions, initially present in the protein layer in a relatively high concentration, cause a local low resistance, thereby increasing the cut-off frequency of the ISFET. The subsequent decrease

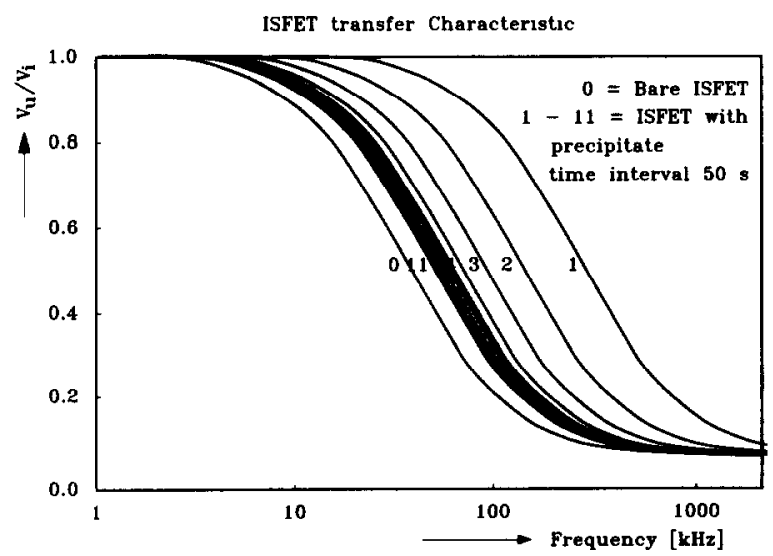

Fig. 8. Transfer characteristic $\mathrm{Nu} / \mathrm{Vi}(f))$ of a bare ISFET (O) and after deposition with $H S A / a H S A$ precipitate $(1-11)$ measured with time intervals of $50 \mathrm{~s}$. of the cut-off frequency with time was explained by outdiffusion of these ions. At equilibrium, when no further change in cut-off frequency was observed, the ionic conduction in the layer was supposed to be still higher than in the bulk solution, resulting in the higher cut-off frequency of the system with respect to that of a bare ISFET. This phenomenon was explained by the Donnan effect, which would be responsible for the additional ions in the layer, being the counterions for the fixed protein charges. The $\mathrm{pH}$ sensitivity of this equilibrium state supports this explanation, because of the $\mathrm{pH}$-controlled charge of proteins.

It may be concluded at this point that a layer of proteins may be sufficiently dense, possibly due to conformational changes, or even denaturation, to explain the observed capacitive changes, but that on the other hand many experimental results show that protein layers may also be very porous. In the latter case the layer shows a specific ionic conductivity which may be related to the state of protein charge, as can be explained with the Donnan effect. Although information can, in principle, also be achieved concerning interfacial potentials from the capacitive measurements with EIS systems, the reported results are difficult to interpret in this respect. Whether proteinrelated interfacial potentials can be measured more obviously in other ways will be considered in the next sections.

\section{THE MEASUREMENT OF PROTEIN- RELATED POTENTIALS}

In the literature concerning immunologically induced electrical potentials, different types of measurements can be distinguished, each based on observed specific phenomena such as charge redistribution, streaming potential, lipid membrane dipole potential and electrode surface potential. The corresponding literature will first be reviewed and evaluated in the following sections, after which the Donnan theory will be treated as a possible explanation for the observed phenomena. Based on the related considerations concerning possible methods of measurement, finally a new approach to immuno sensing will be introduced and described in the last section.

\section{CHARGE REDISTRIBUTION}

It is nowadays generally accepted that under normal physiological conditions the intrinsic 
charge of a protein cannot be measured directly with a potential sensor, because of the screening effect of the counterions. This does not mean, however, that charge redistribution, as a timeconfined process due to an immuno reaction at an antibody- or antigen-coated electrode, cannot be measured, as is shown by Katsube \& Hara (1987). They used two iridium oxide electrodes, both coated with IgG antigen by means of an LB technique, while the antigen was inactivated at one of the electrodes by exposure to UV light. The two electrodes were connected to each other by means of a resistor with a relatively low value (order of $10 \mathrm{k} \Omega$ ). This set-up is schematically given in Fig. 9(a). Upon injection of IgG into the solution in which the two electrodes were immersed, a current transient occurred due to a charge redistribution at the active electrode, as shown in Fig. 9(b).

The IgG antibody concentration could be determined from the time integral of the current transient $(\Delta Q)$.

It is confusing that the authors denoted their sensor as a potentiometric sensor, most probably because they measured a voltage over a resistor. It should be stated, however, that the actual output of the sensor is a temporal current, due to a charge redistribution. This may also generate a potential at the electrode side, but this potential is not the actual measurand.

\section{STREAMING POTENTIAL}

The contact between a solid surface, for instance an electrode, and an electrolyte solution creates an electrical double layer with a diffuse layer of ions in the electrolyte. If the electrolyte solution is

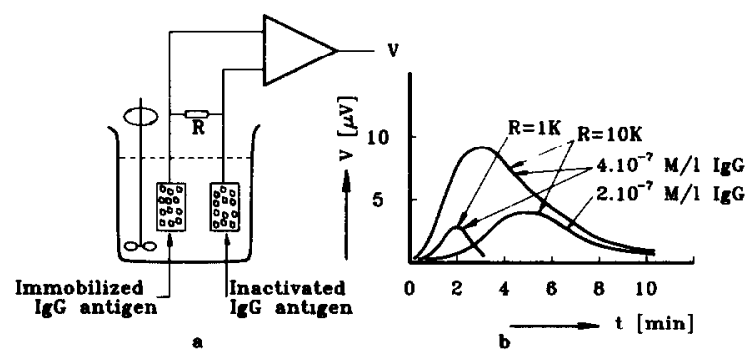

Fig. 9. (a) Two Irox electrodes, one deposited with active IgG antigen and one with inactivated IgG, connected together with a resistor $\mathbf{R}$ (b) Current transient, measured with the circuit of Fig. Y(a), upon the addition of IgG. (Redrawn from Katsube \& Hara (1987).) caused to flow relative to the solid surface, part of the diffuse layer will be disturbed, resulting in a potential difference in the flow direction. This potential is called a streaming potential. The concentration, conductivity, viscosity and dielectric constant of the solution will influence the value of the streaming potential, as well as the presence of molecules, adsorbed to the solid surface. This electrokinetic phenomenon can be made useful for measuring specific interactions between the adsorbed molecules and corresponding anti molecules in the streaming solution. Glad et al. (1986) observed a correlation between the streaming potential and the carbohydrateinduced desorption of Concanavalin $\mathrm{A}$ in a tube which was filled with partially hydrolyzed Sephadex G 50. The measured potentials are, however, very small (parts of $\mathrm{mVs}$ ) and the measurement set-up is not very practical in view of easy-to-use biosensors.

\section{LIPID MEMBRANE DIPOLE POTENTIAL}

The investigation of lipid membranes with incorporated proteins originates from the ambition to mimic biological membranes, such as the postsynaptic membrane in neurons, containing receptor molecules which are sensitive to, for example, acetylcholine, as schematically drawn in Fig. 10.

For an imitation of such a membrane, the similarity is most optimal if a bilayer lipid membrane (BLM) with incorporated biomolecules is stretched as an artificial biomembrane between two electrolyte batches (Thompson \& Dorn, 1988). These membranes are usually made as a symmetrically oriented double layer of lipid molecules on a porous support. If properly made, which, however, appears to be difficult, closepacked and aligned arrays of molecular dipoles should produce a so-called surface dipole potential. If receptor molecules are incorporated

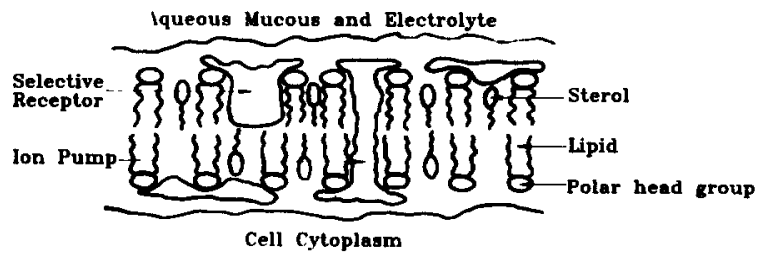

Fig. 10. Schematic representation of natural membrane. which should be imitated. 
in a BLM membrane, electrochemical experiments can be performed, mostly concerning the measurement of ion-channel currents in the order of $\mathrm{pA}$ upon stimulation with specific chemical agents to which the receptor appears to be sensitive.

In order to construct a practical chemoreceptive BLM sensor, the related research has been moved to the construction of BLM layers on a solid substrate by means of the Langmuir Blodgett (LB) technique (Moriizumi, 1988). Apart from the ambition to develop a new type of biosensor in this way, the LB technique, as such, is also used to investigate the intrinsic dipole moment of lipid molecules and the modulation of the related surface potential in the case of selective bimolecular systems. For these experiments the surface potentials have to be measured at an air-water interface, containing the LB layer in an LB trough, by means of a non-contacting electrostatic voltmeter (vibrating reed). In this way transient dipole potentials in the order of 20$80 \mathrm{mV}$ with a duration of less than $1 \mathrm{~s}$ have been measured upon the formation of antibodyantigen and Concanavalin A-saccharide pairs, which were injected subsequently in the water subphase (Thompson et al., 1981). Although it must be stated that the explanation of the observed phenomena is still in discussion and the experimental set-up is far from practically applicable as a sensor, the results clearly show that during an immnochemical or similar reaction electrical charges will be redistributed which may result in measurable potential or current transients.

Because the conditions of the BLM experiments as mentioned above are not so practical in terms of a realistic sensor, attempts have been made to develop less sophisticated measuring devices. A few papers have already dealt with more concrete sensors, in the form of biomimetric silicon chips (Hongyo et al., 1987; Toko et al., 1987; Hayashi et al., 1989). In these cases, however, the silicon was only used as a substrate for an electrode on which a gel and a BLM membrane were attached, or as a substrate with micropores (which can be made very small and reproducible in silicon), over which a bilayer was deposited. Also in these experimental sensors electrical potentials have been measured, sometimes oscillatory, influenced by chemical agents such as taste substances. A clear explanation of the observed phenomena is, however, not given. It is only suggested that conformational changes in membrane constituents play an essential role.

Summarizing the experimental results of the BLM-based experiments, it may be concluded that in one way or another conformational changes of the biomolecules may lead to alterations in membrane potentials, but also that ion permeability may occur, even at the level of one (channel) molecule only. The fact that already one protein molecule may induce a local conductivity can result, however, in serious problems with respect to potentiometric sensors which are constructed explicitly on the basis of a whole layer of proteins. Nevertheless, potentiometric sensors have been investigated in various ways, as will be summarized in the next section.

\section{ELECTRODE SURFACE POTENTIAL}

The idea of an immunoelectrode, measuring potentiometrically a change in surface charge at a hydrophobic membrane deposited on a metal electrode was introduced by Janata (1975). This change was expected to be induced by an immunochemical reaction, occurring at the membrane surface. To illustrate the effect, a nonimmuno model system was used. Concanavalin A was covalently attached to the surface of a PVC membrane, deposited on a platinum wire. The electrode potential responded strongly upon the addition of yeast mannan, which is known to react with Con $\mathrm{A}$, forming a strong complex. A typical registration is shown in Fig. 11.

No response was measured when Con A was immobilized on the surface of a $\mathrm{pH}$ glass

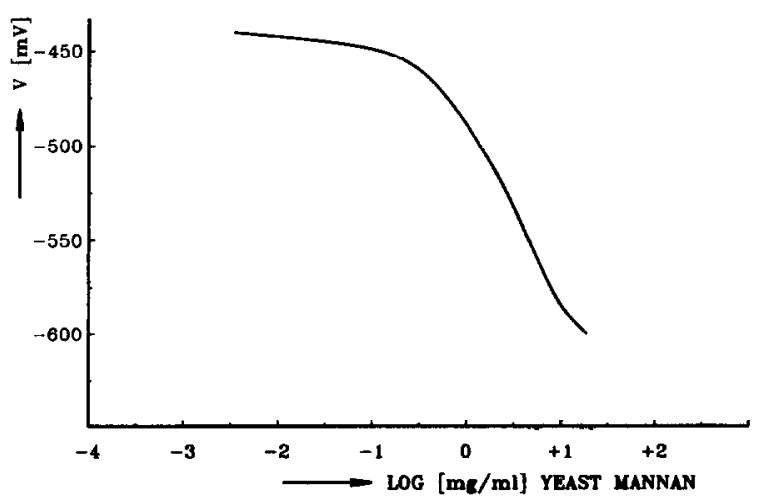

Fig. 11. Dependence of the potential of Con A immunoelectrode on concentration of yeast mannan against SCE. (Redrawn from Janata (1975).) 
electrode, in which case the electrode potential was, according to Janata (1975), predominantly determined by the $\mathrm{pH}$ of the sample solution. This led to the conclusion that, for this type of affinity electrodes, only those membranes can be used which have a minimal interaction with water.

Although these first experimental results were later seriously criticized by the author himself (Collins \& Janata, 1982; Janata, 1986), the idea of constructing potentiometric membrane-type immunosensors, which derive their selectivity from the chemical affinity of an antigen for its corresponding (membrane-bound) antibody, has been described in the literature at regular intervals since Janata's first illustration.

An immunosensor was developed for the determination of syphilis antibody in human sera by Aizawa et al. (1979), for IgG by Aizawa (1978) and for hCG by Yamamoto et al. (1978). It is striking that in these cases only potential changes were measured in the order of a few $\mathrm{mV}$ 's over the full range of analyte concentrations, whereas Janata showed a curve from which a response in the order of $80 \mathrm{mV}$ per decade can be deduced in the sensitive area (see Fig. 11). Collins \& Janata (1982) gave a critical evaluation of the mechanism of potential response of antigen polymer membranes. They described and discussed potentiometric measurements, made on FET devices, provided with a PVC membrane containing a mixture of cardiolipin, lecithin and cholesterol. These PVC/antigen membranes appeared to respond to some specific proteins by changing the surface potential. It was, however, concluded that this is not a direct measurement of immunologically induced dipole potentials, but rather an indirect measurement of a disturbed membrane potential, which originates from the permselectivity of a PVC membrane. Permselective behaviour of PVC is a known phenomenon, as well as the intrinsic conductivity of these membranes. The fact that membranes with a thickness in the order of $10 \mu \mathrm{m}$ on top of an ISFET still result in a stable operation of these devices proves also that PVC membranes are conductive as compared to the usual gate insulators. Therefore it was concluded by Collins and Janata (1982) that the observed proteininduced potential changes are caused by modulation of the ion exchange process at the interface of the membrane and are thus a secondary phenomenon, while the primary response is due to small inorganic ions, such as sodium ions.

Whether the explanation given above is also valid for the results of the other experiments described above is difficult to prove, although Aizawa et al. (1977) also related the antigenic potential response to changes in the anionic transport number. The disturbance of the potential of an ionselective electrode upon adsorption of proteins is mentioned explicitly in the papers of Solsky and Rechnitz (1979) and Keating and Rechnitz (1985). A problem of these electrochemical immunoassays is, however, the inherent interference with the primary ion to which the electrode responds. This problem can be circumvented if a second ISE, not affected by any protein adsorption, can be used as a reference electrode in a differential set-up.

In some recent papers Gotoh et al. $(1987,1989)$ reported on an acetylcholine and a human serum albumin (HSA) sensor, based on an ISFET with a polyvinyl butyral membrane. The hydrophilic PVB membranes were hand-casted on the ISFET chips and had a thickness in the order of $25 \mu \mathrm{m}$ and a water content of $27.6 \%$. Two types of acetylcholine sensors have been constructed, one with the acetylcholine receptor directly fixed to the PVB membrane and the other with the receptor embedded in a lipid layer of lecithin. All measurements were made with respect to a reference ISFET. Both devices responded to acetylcholine, but with a different sensitivity (Fig. 12).

The sensor with the lipid layer provided about $2 \mathrm{mV}$ of gate voltage upon injection of $10 \mu \mathrm{M}$

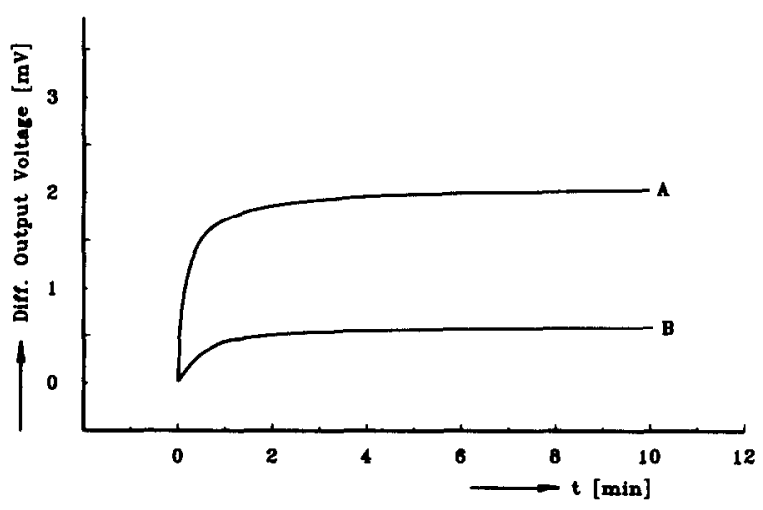

Fig. 12. Response curves of PVB electrode on acethylcholine. (A), acethylcholine receptor embedded in a lipid membrane covering the PVB membrane; $(B)$, receptor directly fixed to the PVB membrane. (Redrawn from Gotoh et al. (1987).) 
acetylcholine, about $1.4 \mathrm{mV}$ larger than that of the sensor without the lipid layer. This difference was thought to be caused by the effect of a sodium ion flux through the acetylcholine channel, which effect should be larger in the case of the lipid layer due to the existence of a sodium gradient over the lipid layer. On the other hand, it is stated by these authors that the positive charge of acetylcholine causes the potential change, unfortunately without any theoretical or other explanation. It is, however, difficult to imagine how both phenomena - the charge effect and the ion channel currents - can exist at the same time in an ion-conducting membrane. Furthermore it should be noted that the observed signal amplitudes are very small and in the order of known interference signals such as drift, instability of the sensor as well as the reference sensor, etc. Therefore the results should be considered with care.

The same critical remarks should be made regarding the experimental results reported in the same papers, concerning an ISFET which was coated with an anti-HSA immobilized PVB membrane. This sensor responded to HSA, in the $m V$ range, which again was explained on the basis of the protein charge. Although in the first instance this effect seems to be present because the sensitivity of the sensor appeared to be a function of $\mathrm{pH}$ - which can be explained as the tuning of the protein charge by the $\mathrm{pH}-$ the effect of the underlying $\mathrm{pH}$-sensitive ISFET upon a change in $\mathrm{pH}$ is totally ignored by the authors. This is difficult to accept, taking into account the gel-like structure of the membrane.

From the papers reviewed in this section it is concluded that the different experimental results are difficult to compare, due to the wide scope of devices used, including the membranes and the biomolecules involved, as well as the differences in experimental conditions such as $\mathrm{pH}$ and ionic strength of the sample solutions. Furthermore, lack of consistency with respect to the explanation of the various experimental results makes comparisons difficult. Terms such as 'mixed potentials', 'charge effects', as well as the existence of ion currents in membranes which are deposited on a solid substrate, without describing the return current paths, indicate a relatively vague understanding of what actually happens at the outer membrane interface, the bulk of the membrane and the inner membrane interface (the ISFET surface). Although a large amount of literature exists concerning steady-state membrane behavior, including membranes with fixed charges, up to now it has been difficult to link the existing theories with the available experimental results. However, a first attempt to describe what may be the matter will be given in the next section.

\section{THE DONNAN EQUILIBRIUM}

Because most of the membranes including protein layers, as mentioned in the previous section, contain a certain amount of water, or are even extremely hydrophilic, and because the measurements described are focused on steadystate conditions, a generic theoretical approach may start with the equilibrium description of two aqueous solutions, (1) and (2), respectively, separated by a thin membrane as represented in Fig. 13.

Suppose that solution (1) contains a dissociable salt, for instance $\mathrm{NaCl}$, while solution (2) also contains $\mathrm{NaCl}$, in which, however, the $\mathrm{Na}^{+}$ions are partly replaced by an equivalent amount of positive charges on protein molecules $\left(\mathrm{p}^{+}\right)$. The membrane is supposed to be permeable for the small cations and anions, but not for the macromolecules. The sodium ions will diffuse through the membrane from solution (1) to solution (2), due to the concentration gradient, initially tracking the chloride ions, even against the developing concentration gradient for chloride ions.

This phenomenon was first recognized by Donnan (Donnan, 1911), who proved that the diffusion of both types of ions will be in equilibrium as soon as the concentration ratios for $\mathrm{Na}^{+}$and $\mathrm{Cl}^{-}$ions $\left(c_{\mathrm{Na}}\right.$ and $\left.c_{\mathrm{Cl}}\right)$ have become reciprocal in the two solutions.

$$
\frac{c_{\mathrm{Na}}{ }^{+}(1)}{c_{\mathrm{Na}}{ }^{+}(2)}=\frac{c_{\mathrm{Cl}^{-}(2)}}{c_{\mathrm{Cl}^{-}(1)}}=r_{\mathrm{D}}
$$

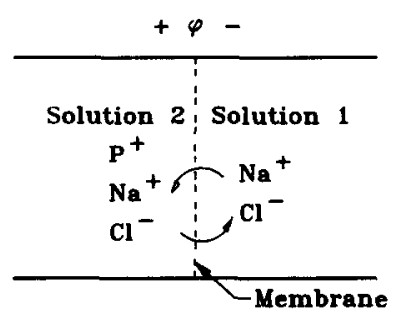

Fig. 13. Schematic representations of Donnan equilibrium. 
where (1) and (2) refer to the solutions (1) and (2), respectively, and $r_{\mathrm{D}}$ is the so-called Donnan equilibrium constant (the activity coefficient is assumed to be unity). The process leads to a difference in osmotic pressure between the two solutions, while in addition an electrostatic potential difference is established over the membrane to prevent a net movement of the ions. This membrane potential exactly balances the concentration gradients of the diffusible ions and its value is given by the Nernst formula

$$
\phi_{\mathrm{D}}=\frac{R T}{F} \ln r_{\mathrm{D}}
$$

Note that the Donnan effect not only creates a membrane potential, but also a redistribution of all diffusible ions between both solutions. This result is in fact caused by the presence of the large protein molecules at one side of the membrane, which cannot diffuse through the membrane.

The bulk electroneutrality for both solutions dictates that

$$
c_{\mathrm{Na}}{ }^{+}(1)-c_{\mathrm{Cl}^{-}(1)}=0
$$

and

$$
c_{\mathrm{Na}^{+}(2)}-c_{\mathrm{Cl}^{-}{ }_{(2)}+c_{\mathrm{P}^{+}}=0}
$$

where $c_{\mathbf{P}}{ }^{+}$is the concentration of protein-fixed positive charges. in

Combination of eqns (3), (4), (5), and (6) results

$$
\begin{aligned}
& c_{\mathrm{Na}^{+}(2)}=\frac{1}{2}\left(\sqrt{4 c_{\mathrm{s}}^{2}+c_{\mathrm{p}}{ }^{2}+}-c_{\mathrm{p}}+\right) \\
& c_{\mathrm{Cl}^{-}(2)}=\frac{1}{2}\left(\sqrt{4 c_{\mathrm{s}}^{2}+c_{\mathrm{p}}{ }^{2}+}+c_{\mathrm{p}}+\right)
\end{aligned}
$$

where $c_{\mathrm{s}}$ is the salt concentration in solution (1) and equals $c_{\mathrm{Na}}+_{(1)}$ as well as $c_{\mathrm{Cl}}-{ }_{(1)}$.

For the Donnan potential $\phi_{\mathrm{D}}$ it follows that

$$
\phi_{\mathrm{D}}=\frac{R T}{F} \ln \left[\frac{\sqrt{4 c_{\mathrm{s}}^{2}+c_{\mathrm{p}}{ }^{2}+}+c_{\mathrm{p}}+}{2 c_{\mathrm{s}}}\right]
$$

From eqn (9) it can be concluded that for $c_{p}+=0$ no Donnan potential will be present and an equal distribution of the ions will occur. The value of $\phi_{\mathrm{D}}$ in the case where $c_{\mathrm{p}}+\neq 0$ depends on the ratio between $c_{\mathrm{p}}+$ and $c_{\mathrm{s}}$. In general it can be stated that, especially in the case of a low ionic strength $\left(c_{\mathrm{s}}<1 \mathrm{~mm} /\right.$ litre $)$, the presence of a moderate amount of protein-fixed charge $\left(c_{\mathrm{p}}+\right)$ will result in a measurable Donnon potential as well as in an adaptation of the bulk concentration of all ions.

The distance over which the Donnan potential is extended at both sides of the membrane is described by the Gouy-Chapman theory, and characterized by the Debye length and thus by the respected ion concentrations in both solutions. The change in the ion concentrations extends over the same distance. Outside this distance (the neutral bulk) the potentials as well as the ion concentrations are constant but different.

It should be noted that in a practical case where solution (1) has a larger volume than solution (2) the bulk concentrations in solution (2) will have been adapted in a measurable amount due to an appreciable Donnan effect, but in solution (1) only, if the relative change of the ion concentration due to the transport to or from solution (2) is high enough.

Concentrating now on the examples of electrodes with adsorbed layers of proteins, as described in the previous sections, the protein layer can be considered as an analogue of solution (2), while solution (1) is the sample solution. The membrane as present in Fig. 13 can now be omitted, because the proteins are adsorbed to the electrode. The electrode itself can be a potential-measuring electrode (noble metal electrode or REFET), or an ion-sensitive electrode, which is of course also potentialsensitive (coated wire ISE, ISFET, membranecovered (HEMFET). The measurand may be adsorption of a protein or an immuno reaction occurring at an antibody-precoated electrode. One can distinguish three different cases, which will be treated subsequently below.

\section{Case (1)}

Assume that the electrode is covered with a layer of proteins, directly adsorbed to the surface, in contact with a salt solution as represented in Fig. 14(a). Furthermore, it is assumed that no protein-free bulk solution is present between the layer and the electrode surface. Due to the adsorption process the concentration of proteins is enlarged at the electrode with respect to that of the sample solution. The Donnan process will manifest itself under the conditions as mentioned above. The protein layer will be at a Donnan potential with respect to the solution and a redistribution of all types of ions will occur, 


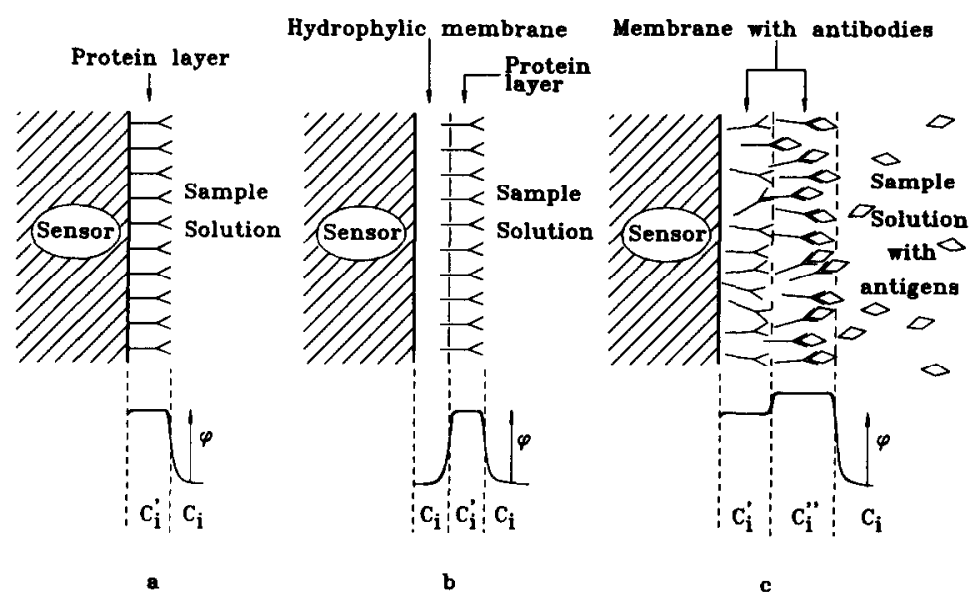

Fig. 14. Schematic representation of potential or ion sensor with (a), directly adsorbed protein layer (case (1)); (b), intermediate hydrophylic membrane (case (2)); (c), antibody containing membrane (case (3)). $\phi$ is the potential and $\mathrm{c}_{i}, \mathrm{c}_{i}^{\prime}$ respectively $\mathrm{c}_{i}^{\prime \prime}$ are the concentrations of all type of ions in the relevant phases.

according to the Donnan ratio and in a measurable amount inside the protein layer.

If the sensor is a potential sensor, the Donnan potential can be measured with respect to an external reference electrode. The reported results with protein-coated wires may operate along this line, although it should be mentioned that a metal wire is not an ideal potential sensor, because it is not in thermodynamical equilibrium and is thus unstable and highly sensitive to interferences. However, in the case of FET devices, a stable REFET could be used to measure the Donnan potential, but no examples of such sensors are described in the literature.

If the sensor is an ion-selective sensor, such as a potassium-sensitive $\mathrm{CHEMFET}$, an $\mathrm{Ag} / \mathrm{AgCl}$ electrode or a $\mathrm{pH}$-sensitive ISFET, the Donnan potential will also be measured as in the previous case. In addition to this, the ion sensor will, however, respond to the ion concentration inside the protein layer, which is changed as a result of the Donnan partition. This response will compensate the effect of the Donnan potential. This compensation will be complete in the case of a sensor which responds with a sensitivity of $59 \mathrm{mV} / \mathrm{dec}$, which is also the sensitivity of the Donnan potential $(2.3 \mathrm{RT} / \mathrm{F})$. If the sensor responds with a smaller sensitivity, for instance an $\mathrm{Si}_{3} \mathrm{~N}_{4}$ ISFET $(45 \mathrm{mV} / \mathrm{pH})$, a small net result of the Donnan potential will be measured. This may be the reason that most of the protein- and immunoFETs in sample solutions with low ionic strength, being the condition for the Donnan effect, show only responses in the order of some $\mathrm{mV}$ 's.

\section{Case (2)}

Assume that the sensor is precoated with a bare hydrophylic membrane, having a partition coefficient of 1 , with a protein layer adsorbed at the membrane/solution interface, or possibly also in the outer surface of the membrane. Now a three-compartment system is created as represented by Fig. 14(b).

The presence of the layer of proteins will now create a symmetrical Donnan effect, with identical potentials at both sides of the protein layer. The result is that no net Donnan potential can be measured, either with a potential sensor or an ion sensor. Inside the protein layer an ion redistribution will take place as well as in the external solution, which in this case consists of the sample solution and the membrane solution, which are in equilibrium with each other. Because in general the volume of the sample solution will be relatively large, the ion redistribution will hardly affect the absolute value of the different ion concentrations, including those inside the membrane. Therefore in the case of an applied ion sensor, no ion response will be measured in contrast to the situation as in case (1). Thus it should be concluded that in case (2) no net potentials can be measured, nor an ionic effect. This result may also be the reason for the disappointing results of some types of biosensors. 
It should be mentioned, however, that if the sample and the membrane volumes are relatively small, the Donnan-induced ionic transport may be able to change the ion concentrations in the membrane in a measurable amount. In that case the ion sensor will thus respond to the presence of the protein layer at some distance from the sensor interface.

Note that in a practical case of a particular protein sensor it will not always be clear whether the sensor will operate as a case (1) or case (2) system.

In cases (1) and (2) it was assumed that the experimental conditions for the development of the Donnan phenomenon were fulfilled. In experiments where $c_{\mathrm{p}}+$ is small with respect to $c_{\mathrm{s}}$, eqn (9) predicts that no Donnan potentials will occur, nor an ion redistribution. This means that in this case, whether it is a case (1) or case (2) system, either with a potential sensor, or an ion sensor, no effect will be measured. Disappointing experimental results may therefore also be caused by non-optimal experimental conditions. In this respect it should be noted that besides the ionic strength of the sample solution and the density of the adsorbed proteins, the type of the protein and the $\mathrm{pH}$ also determine the success of a measurement.

\section{Case (3)}

It may be concluded from the two cases considered above, that when the required experimental conditions are fulfilled the biggest signal can be expected from a case (1) system. This also counts for a sensor which is coated with a membrane containing homogeneously immobilized proteins. If in this case an immuno reaction takes place, because the electrode is precoated with an antibody-containing membrane and the sample solution contains the corresponding antigens, it may appear that immuno complexes are only formed in the outer part of the membrane. The result will be an asymmetrical Donnan effect, as schematically represented in Fig. 14(c). The result is that a case (1) system thus develops the characteristics of a case (2) system with respect to the second protein, whose presence is therefore not measured. To prevent this loss in sensitivity, one therefore should take care that the membrane is porous enough to provide the immuno reaction throughout the whole membrane. Only then can the operation as a case 1 system be guaranteed.
In addition to the effects considered in the three typical cases, it should be noted that the use of a reference electrode in the protein-containing sample solution may also result in a liquid junction potential with similar effects as described above, causing deviations from the reference electrode potential. Therefore it is recommended that the measurements should be carried out as differential measurements. This was, however, not always the case in the experiments reported in the literature and summarized in the previous section.

In spite of the possibilities of operational mechanisms described above, the reported small effects in the $\mathrm{mV}$-range, described and discussed in the previous section, can still not be easily explained. If the reported responses are not caused by sensor-related artefacts such as drift, or by a changing reference electrode potential, small potentials can be the result of a relatively small Donnan effect, related to the ratio $c_{\mathrm{p}}+/ c_{\mathrm{s}}$, or by larger Donnan effects which are, however, partly compensated by local changes in the ion concentrations. Therefore it is not possible to explain the reported results unambiguously. It should, however, be noted that the considerations given in this section do not need any description in the form of obstruction of ion diffusion effects at ion-selective sensors, resulting in 'mixed' potentials, whatever this may be. Nor is the formation or blocking of ion channels involved, which is in any case difficult to interpret because of lack of return current paths for flowing ion currents.

As a final conclusion it should be stated that the development of an immuno electrode for the direct monitoring of an immuno reaction is more difficult than originally thought. The exploitation of the Donnan effect may be a solution, but a drawback is the limitation to low ionic strength solutions. Furthermore, it will always be difficult to measure small static potentials without problems of drift. Therefore we decided in our group to follow a completely different route for the construction and application of an immuno sensor, as will be dealt with in the next section.

\section{A NEW APPROACH TO IMMUNO SENSING}

Schasfoort et al. $(1989 b, 1990 a)$ used a porous membrane of polystyrene beads $($ ( $120 \mathrm{~nm})$, cross-linked with agarose on top of an ISFET, 
having a thickness of approximately $5 \mu \mathrm{m}$. All types of ions and proteins are free to move inside the membrane pores, while the proteins will adsorb to the polystyrene particles. Due to the fact that the ISFET has a $\mathrm{Ta}_{2} \mathrm{O}_{5}$ gate, with a sensitivity of $56-57 \mathrm{mV} / \mathrm{pH}$, Donnan potentials which may possible form are completely compensated by a shift in internal membrane $\mathrm{pH}$, as described in case (1) above. Under conditions of high ionic strength, also no response can be expected. Indeed no static response to protein adsorption could be measured for low as well as high ionic strength, completely in accordance with theoretical considerations as mentioned in the previous section. The new approach in the type of operation as introduced by Schasfoort $e t$ al., however, is that no longer static, but on the contrary dynamic measurements are carried out. After establishment of an equilibrium state in a salt concentration of $10 \mathrm{mM} \mathrm{KCl}$ at a certain $\mathrm{pH}$, a sudden change in the $\mathrm{KCl}$ concentration is induced (to $40 \mathrm{mM}$ ) and the response to this 'ion step' is measured. Thus instead of measuring static potentials, the potential change between two equilibrium states is measured, resulting from an induced disturbance.

Due to the difference in mobility of cations and anions inside the membrane, influenced by the internal protein-related charge density, a transient membrane diffusion potential will be created, which is measured by the ISFET. Because proteins are amphoteric molecules, the protein charge can be changed by the $\mathrm{pH}$ of the solution. Thus by slowly changing the $\mathrm{pH}$ of the solution while carrying out the ion step procedure with regular intervals, transient membrane potentials with an amplitude $\phi_{m}$ can be measured as a function of the $\mathrm{pH}$ and thus as a function of the protein charge. Figure 15 shows a typical result of such a registration, in which case lysozyme has previously been adsorbed to the polystyrene beads.

As can be seen in Fig. 15, at a certain pH no response at all is observed. This $\mathrm{pH}$ is called the inversion point, $p \mathrm{I}^{\prime}$, of the membrane, at which the net charge density in the membrane (protein charge and intrinsic membrane charge) is zero.

Figure 16 shows a set of $\phi \mathrm{m} / \mathrm{pH}$ curves when the membrane was loaded with various concentrations of HSA. It can be seen that there is not only a shift in the inversion point $p \mathrm{I}^{\prime}$, but also an increase in the slope of the curves with increasing protein concentration. The hinge-point of the

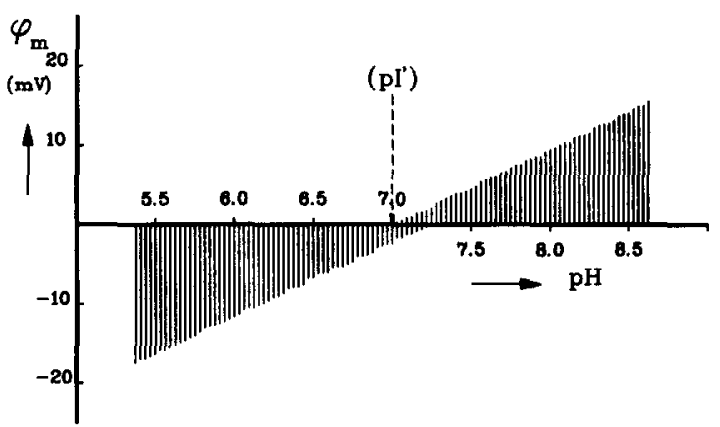

Fig. 15. Registration of transient potentials, caused by the ion step for adsorbed lysozyme as a function of the $p H$ of the solution. $\mathrm{pI}^{\prime}$ is denoted as the inversion point of the transient polarity.

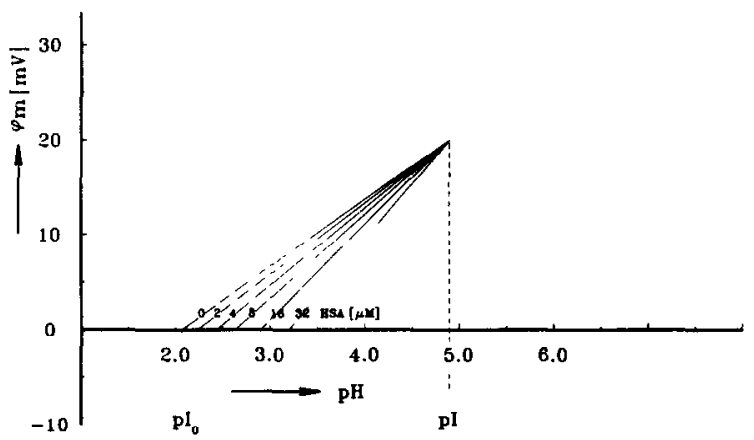

Fig. 16. $\phi_{m} / p H$ curves for different concentrations of HSA in the membrane. $\mathrm{pI}_{0}$ is the inversion point of the bare membrane: $\mathrm{pI}$ is the isoelectric point of HSA.

curves appears to be the isoelectric point, $p I$, of HSA $(p I=4 \cdot 9)$.

An immuno reaction can be monitored by first adsorbing the antibody inside the membrane, followed by an incubation with the corresponding antigen, or the other way around. Figure 17 shows the result of an HSA/ $\alpha$ HSA experiment with increasing $\alpha \mathrm{HSA}$ concentration $(0.06-64 \mu \mathrm{M})$ and incubation time, using a precoated HSA immunoFET.

It can be seen in Fig. 17 that the antibody $\alpha$ HSA is able to change the inversion point $p \mathrm{I}^{\prime}$ of the HSA-coated membrane from $3 \cdot 70$ to 5.55 .

In addition to the experiments as described above, it has been shown that the ion step procedure is also applicable for the detection of uncharged species, if these are provided with a charge label (Schasfoort et al., 1990b). Preliminary experiments show that progesterone can be detected by using competitive binding with a charged progesterone-lysozyme conjugate in the 


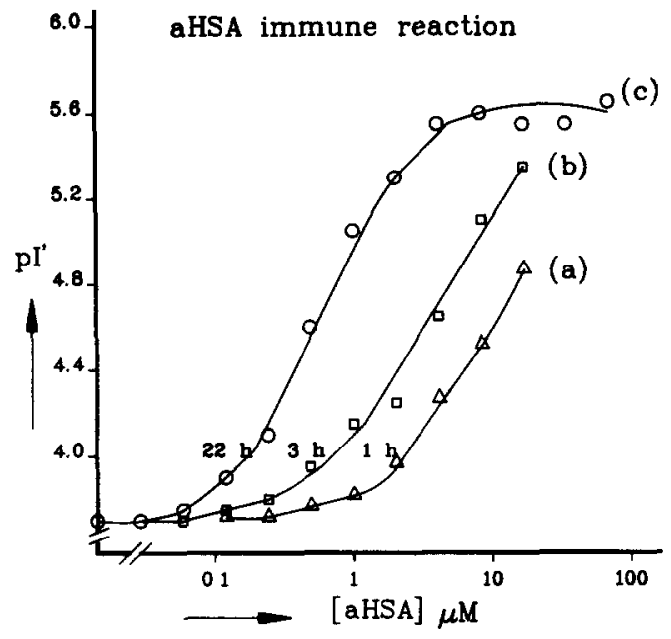

Fig. 17. Detection of the $\alpha H S A / H S A$ immuno reaction by measuring the inversion point $\mathrm{pI}$ ' as a function of the aHSA concentration in the solution. Experimental results after (a).

I hour; (b), 3 hours, and (c) 22 hours of incubation.

membrane. A detection limit of $10^{-8} \mathrm{M}$ could be achieved.

The ion step experiments show that the dynamic use of immuno electrodes opens a new way for the direct measurement of charge densities in membranes and thus of adsorbed proteins or protein complexes. The operational mechanism is based on the modulation and measurement of ion mobilities. In a practical situation the experimental conditions should of course be adapted to the specific immuno reaction to be monitored. In this case an optimal $\mathrm{pH}$ should be chosen, as well as an adequate electrolyte and amplitude of the ion step, at which the precoated membrane should produce a clear transient membrane potential. After the incubation with the antibody or antigen to be measured, the same ion step should produce a large deviation from the first transient, if possible with the opposite sign.

\section{CONCLUSION}

The results of most impedance and potentiometric measurements, aimed at the direct detection of an immuno reaction occurring at the surface of an electrode, have until now been disappointing and, moreover, difficult to explain. The results of some experiments reviewed in this paper can possibly be explained in the light of the Donnan phenomenon, but the experimental conditions should be varied ( $\mathrm{pH}$, ionic strength) to come to a definite conclusion.

The poor results of static measurements are overshadowed by the promising results of the dynamic measurements on porous protein-coated membranes, as described in the last section. These exhibit large transient potentials which can be explained by the modulation of ion mobilities inside a layer of proteins. In addition this type of measurement is independent of any long-term drift of the applied sensor, which is a large drawback for any static measurement.

It is possible that the preliminary results of Katsube \& Hara (1987) as illustrated in Fig. 9 are in fact also the result of a dynamic disturbance of an equilibrium state and are thus related to the modulation of ion mobilities induced by the sudden addition of antibodies.

\section{ACKNOWLEDGEMENTS}

Writing on the subject treated in this paper was greatly stimulated by the combined supervision of PhD students with the author's colleague Jan Greve. Common discussions with these students, Richard Schasfoort, Jan Eijkel and Jans Kruise, were very helpful in judging the literature and evaluating my own opinions. I am most grateful to Gerrit Bultstra for providing the figures.

\section{REFERENCES}

Aizawa, M., Kato, S. \& Suzuki, S. S. (1977). Immunoresponsive membrane, J. Membrane Sci., 2, 125-32.

Aizawa, M. (1978). Electrochemical determination of IgG with an antibody bound membrane. J. Membrane Sci., 4, 221-8.

Aizawa, M., Suzuki, S., Nagamura, Y., Shinohara, Y. \& Shiguro, R. I. (1979). An immunosensor for syphilis, J. Solid-Phase Biochem., 4, 25-31.

Arwin, H., Lundström, I. \& Palmquist. A. (1982). Electrode adsorption method for determination of enzymatic activity, Med. \& Biol. Eng. \& Comp.. 20, $362-74$.

Bataillard, P., Gardies, F., Jaffrezic-Renault. N. \& Martelet, C. (1988). Direct detection of immuno species by capacitance measurements, Anal. Chem., 60, 2374-9.

Bergveld, P. \& Sibbald, A. (1988). Comprehensive Analytical Chemistry, Vol 23. Analytical and Biomedical Applications of Ion-selective Field-effect Transistors, Elsevier/Wilson \& Wilson, Amsterdam. 
Champagne, G. Y., Bélanger, D. \& Fortier, G. (1988). A new electrochemical approach for biosensor design, In Biotechnology Research and Applications, ed. J. Gavora, Elsevier Applied Science, London, pp. 175-84.

Collins, S. \& Janata, J. (1982). A critical evaluation of the mechanism of potential response of antigen polymer membranes to the corresponding antiserum, Anal. Chim. Acta, 136, 93-9.

Donnan, F. G. (1911). Theorie der membrangleichgewichte und membranpotentiale bei vorhanden sein von nicht dialysierenden Élektrolieten. Ein Beitrag zur Physikalisch-Chemischen Physiologie. Zeitschrift für Elektrochemie, Bd. 17, 572-81.

Eddowes, M. J. (1987). Direct immunochemical sensing: basic chemical principles and fundamental limitations, Biosensors, 3, 1-15.

Gardies, F., Martelet, C., Colin, B. \& Mandrand, B. (1989). Feasibility of an immunosensor based upon capacitive measurements, Sensors \& Actuators, $17,461-4$.

Glad, C., Sjodin, K. \& Mattiasson, B. (1986). Streaming potential - a general affinity sensor, Biosensors, 2 , 89-100.

Gotoh, M., Tamiya, E., Momoi, M., Kagawa, Y. \& Karube, I. (1987). Acetylcholine sensor based on ion sensitive field effect transistor and acetylcholine receptor, Anal. Lett., 20, 857-70.

Gotoh, M., Tamiya, E. \& Karube, I. (1989). Micro-FET biosensors using polyvinylbutyral membrane, J. Membrane Sci., 41, 291-303.

Grattarola, M., Cambiaso, A.. Cenderelli, S. \& Tedesco, M. (1989). Capacitive measurements in electrolyteinsulator-semiconductor (EIS) systems modified by biological materials, Sensors \& Actuators, 17, 451-9.

Hayashi, K., Yamafuji, K., Toko, K., Ozaki, N. \& Yoshida, T. (1989). Effect of taste substances on electric characteristics of a lipid cast membrane with a single pore, Sensors \& Actuators, 16, 25-42.

Hongyo, K, Joseph, J., Huber, R. J. \& Janata, J. (1987). Integrated silicon biosensor based on supported phospholipid bilayer membrane, Proc. Transducer '87, Tokyo. Institute of Electrical Engineers of Japan, pp. 808-11.

Ivarsson, B., Jönsson, U., Lundström, I. \& McQueen, D. (1981). Initial protein adsorption on biocompatible metal surfaces, Internal report: Biophysics and medical technology, Linköping University Sweden, LAPPH 81-1.

Janata, J. (1975). An immunoelectrode, J. Amer. Chem. Soc., 97, 2914-6.

Janata, J. (1986). Chemical selectivity of field effect transistors, Proc. 2nd Int. Meeting on Chemical Sensors, Bordeaux, pp. 25-31.

Katsube, T. \& Hara, M. (1987). High sensitive biosensor with a junction FET, Proc. Transducer '87, Tokyo. Institute of Electrical Engineers of Japan, pp. 816-9.
Keating, M. Y. \& Rechnitz, G. A. (1985). Potentiometric digoxin antibody measurements with antigenionophore based membrane electrodes, Anal. Chem. 56, 801-6.

Moriizumi, T. (1988). Langmuir-Blodgett films as chemical sensors, Thin Solid Films, 16, 413-30.

Newman, A. L., Hunter, K. W. \& Stanbro, W. D. (1986). The capacitive affinity sensor: a new biosensor, Proc. 2nd Int. Meeting on Chemical Sensors, Bordeaux, pp. 596-8.

Schasfoort, R. B. M., Streekstra, G. J., Bergveld, P., Kooyman, R. P. H. \& Greve, J. (1989a). Influence of an immunological precipitate on de and ac behaviour of an ISFET, Sensors \& Actuators, 18, 119-29.

Schasfoort, R. B. M., Bergveld, P., Bomer, J., Kooyman, R. P. H. \& Greve, J. (1989b). Modulation of the ISFET response by an immunological reaction. Sensors \& Actuators. 17, 531-5.

Schasfoort, R. B. M., Kooyman, R. P. H., Bergveld, P. \& Greve, J. (1990a). A new approach to ImmunoFET operation, Biosensors \& Bioelectronics, 5, 103-24.

Schasfoort, R. B. M., Keldermans, C. E. J. M., Kooyman, R. P. H. Bergveld, P. \& Greve, J. (1990b). Competitive immunological detection of progesterone by means of the ion-step induced response of an ImmunoFET, Sensors \& Actuators, B1, 368-72.

Solsky, R. L. \& Rechnitz, G. A. (1979). Antibodyselective membrane electrodes, Science, N.Y., 204, 1308-9.

Stenberg, M., Arwin, H. \& Nilsson, A. (1979). Siliconsilicon dioxide as an electrode for electrical and ellipsometric measurements of adsorbed organic molecules, J. Colloid \& Interface Sci., 72, 255-64.

Thompson, M. \& Dorn, W. H. (1988). Selective chemical transduction based on chemoreceptive control of membrane ion permeability, In Chemical Sensors, ed. T. E. Edmonds. Blackie, Glasgow, Chapter 7.

Thompson, M., Wong, H. E. \& Dorn, W. H. (1981). The Langmuir-Blodgett monolayer dipole potential: a smeared dipole model for a lipid array and pulsing of the potential by direct subphase infusion of immunochemical and lectin/polysaccharide complexes, Anal. Chim. Acta, 200, 319-32.

Toko, K. Hayashi, K., liyama, S. \& Yamafuji, K. (1987). Taste sensing system utilizing dynamic properties of phase transition in synthesized lipids, Proc. Transducer '87, Tokyo. Institute of Electrical Engineers of Japan, pp. 793-6.

US Patent Feb. 7, 1978, 4,072,576.

Yamamoto, N., Nagasawa, Y., Sawai, Y., Suda, M. \& Tsubomura, T. H. (1978). Potentiometric investigations of antigen-antibody and enzyme-enzyme inhibitor reactions using chemically modified metal electrodes, J. Immunol. Method., 22, 30917. 